\title{
Mitigation of strong electromagnetic pulses on the LMJ-PETAL facility
}

\author{
M. Bardon $\odot,{ }^{1,}{ }^{*}$ B. Etchessahar, ${ }^{1, \dagger}$ F. Lubrano, ${ }^{1}$ S. Bazzoli, ${ }^{2}$ M. Ferri,${ }^{1}$ J. Ribolzi,${ }^{1}$ P. Mirabel,,${ }^{1}$ A. Compant La Fontaine $\odot,{ }^{2}$ \\ N. Mallejac, ${ }^{3}$ S. Cadra, ${ }^{3}$ L. Chaigne, ${ }^{3}$ S. Depierreux,${ }^{2}$ J. Baggio, ${ }^{1}$ N. Blanchot,${ }^{1}$ G. Birindelli, ${ }^{4}$ \\ A. Casner (1), ${ }^{4}$ and V. T. Tikhonchuk $\mathbb{B}^{4,5}$ \\ ${ }^{1}$ CEA, DAM, CESTA, 33114 Le Barp, France \\ ${ }^{2} C E A, D A M, D I F, 91680$ Bruyères Le Chatel, France \\ ${ }^{3} C E A, D A M, L R, 37260$ Monts, France \\ ${ }^{4}$ CELIA, University of Bordeaux, CNRS, CEA, 33405 Talence, France \\ ${ }^{5}$ ELI-Beamlines, Institute of Physics, Czech Academy of Sciences, 25241 Dolní Břžany, Czech Republic
}

(Received 28 November 2019; accepted 2 September 2020; published 28 September 2020)

\begin{abstract}
Electromagnetic pulses (EMP) present a serious threat for operation of high-power, high-energy laser facilities. Here, we present an efficient strategy for EMP mitigation with a resistive and inductive holder, which is supported by extended numerical simulations and validated in dedicated experiments at the kilojoule/picosecond (kJ/ps) Petawatt Aquitaine Laser (PETAL) facility. Moreover, we demonstrate how a combination of PETAL with the tens of kJ/ns Laser MegaJoule (LMJ) beams may suppress the EMP emission. This method opens another efficient way for the EMP control on high-power, high-energy laser facilities.
\end{abstract}

DOI: 10.1103/PhysRevResearch.2.033502

\section{INTRODUCTION}

LMJ-PETAL [1] is a large-scale laser facility which combines high-energy nanosecond laser beams and a high-power picosecond beam. The LMJ beams [2,3] have energies of several kilojoules each and pulses of several nanoseconds (in 2019, 56 LMJ beams could be focused on target), and the PETAL beam [4] has an energy of $1 \mathrm{~kJ}$ (limited at $400 \mathrm{~J}$ during the 2017-2019 period) and a pulse duration of 0.5 ps. The PETAL beam is employed in high-energy-density science experiments as a source of secondary protons or $\mathrm{x}$ rays for target radiography.

The interaction of a petawatt-class laser pulses with solid targets produces intense electromagnetic fields (EMP) [5-8], which may exceed $1 \mathrm{MV} / \mathrm{m}$, leading to equipment failures, diagnostics damage, and spurious signals in detectors. As part of the PETAL project, we have studied the EMP generation mechanisms in order to predict their impact on the diagnostics and security equipment at the LMJ-PETAL facility. A target charging model [9-11] explains the process of EMP generation in short-pulse laser-plasma experiments by the discharge current neutralizing the charge accumulated on the target on a ns timescale. An intense laser pulse focused on the target surface creates energetic electrons that partially escape and leave a positive charge on the target. A discharge current is induced

\footnotetext{
*matthieu.bardon@protonmail.com

†bertrand.etchessahar@cea.fr

Published by the American Physical Society under the terms of the Creative Commons Attribution 4.0 International license. Further distribution of this work must maintain attribution to the author(s) and the published article's title, journal citation, and DOI.
}

through the target holder after the end of the laser pulse. The holder acts as a dipole antenna, creating a strong electromagnetic signal. A multiphysics and multiscale simulation chain has been developed [9] and validated in several experimental campaigns on different laser facilities. The core of the simulation chain is the SOPHIE code [12], a three-dimensional, particle-in-cell (PIC) code, which solves in a self-consistent manner Maxwell equations for the electromagnetic field and dynamic equations for the relativistic charged particles in the vacuum chamber with appropriate boundary conditions.

\section{EMP MITIGATION STRATEGY}

In the present paper, we describe a mitigation strategy developed for safe PETAL operation, alone and in combination with a multi-kJ ns laser facility LMJ. It is demonstrated that by controlling the target holder impedance, one can reduce the intensity of the EMP by an order of magnitude. Moreover, a joint operation of PETAL and LMJ offers an unexpected possibility of efficient suppression of EMP by appropriate timing of ps and ns laser pulses.

Protection of a laser facility such as the LMJ-PETAL from giant EMPs implies that the level of electromagnetic field at the position of equipment is lower than the susceptibility threshold. Its value varies from $1 \mathrm{kV} / \mathrm{m}$ for electronics to $5 \mathrm{kV} / \mathrm{m}$ for industrial automates and to $25 \mathrm{kV} / \mathrm{m}$ for electro-mechanical devices. These values are deduced from experimental studies conducted with a high-field pulsed generator. The protection strategy is based on a dual approach: shielding the vulnerable devices and mitigation of the EMP emission. For exceptionally high EMPs, the required attenuation could be as high as $70 \mathrm{~dB}$, so that both strategies shielding and mitigation are necessary. 


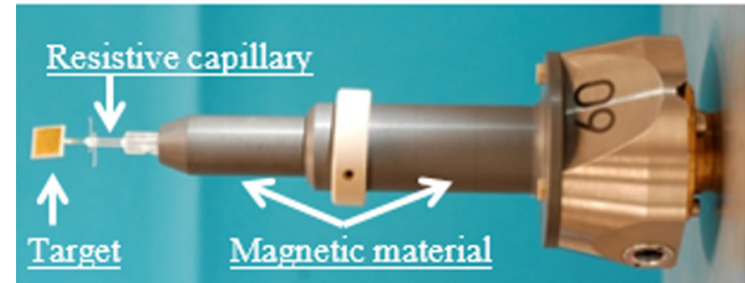

FIG. 1. Photo of the new target holder for mitigation of the EMP emission. The target holder is horizontally oriented in the chamber. The whole length of this holder is $18 \mathrm{~cm}$.

The main idea of the mitigation strategy consists in inhibition of EMP emission by suppressing the return current through the target holder. A new target holder [13] was designed with the help of numerical simulations, fabricated, and tested. It is composed of a glass capillary with a resistive gel inside. The resistance between the two ends of the capillary is about $5-10 \mathrm{k} \Omega$. It is adjusted by addition of $\mathrm{NaCl}$ in the solution. One end of this capillary is attached to the target and the other end is connected to a conducting cylinder surrounded by a magnetic material (ferrite), which operates as an external inductance. The new holder and the target are shown in Fig. 1. It delays the growth of the return current induced by the target charge and limits EMP generation. This holder design was tested at different laser energies ranging from $0.1 \mathrm{~J}$ on the ECLIPSE laser at the Centre Lasers Intenses et applications (CELIA) [14], followed by 80-J campaign at the LULI2000 facility [15] and the first PETAL experiment at $400 \mathrm{~J}$.

\section{NEW TARGET HOLDER PERFORMANCE TEST ON THE LULI2000 FACILITY}

The performance of the new resistive target holder in the experiment on the LULI2000 facility is demonstrated in Fig. 2. Compared to the reference conducting holder, the new holder with a resistance of $10 \mathrm{k} \Omega$ reduced the discharge current by a factor of 30 [Fig. 2(a)] and the amplitude of the magnetic field detected with B-dot probes by a factor of 3 in the frequency range of $0.5-3 \mathrm{GHz}$ [Figs. 2(b) and 2(c)]. The difference between the mitigation factors of current and field can be explained as follows. First, the discharge current was measured at the bottom of target holder, near the ground. The current intensity near the target is higher because the magnetic inductance operates over the whole length of the holder. Second, the target holder is the main source of EMP but it is not the only one; other processes may also contribute to the EMP emission.

In the LULI campaign, we have also tested the performance of the insulating holder (glass capillary). For the unfocused shots, where the intensity was about $10^{18} \mathrm{~W} / \mathrm{cm}^{2}$, we observed a clear reduction of the discharge current and the radiated magnetic field with both holders [cf. Fig. 3(a)]. But for the focused shots, where the intensity was greater than $10^{19} \mathrm{~W} / \mathrm{cm}^{2}$, the insulating holder showed a discharge current intensity quite similar to the reference conducting holder [cf. Fig. 3(b)]. No reduction of the radiated magnetic field was observed either. That means that at high laser intensity shots, electrical breakdown along the glass capillary surface takes place, which is not the case for the resistive holder. This observation motivated us to use in the first PETAL experiments only a holder with the resistive part in order to reduce the risk of electrical breakdowns.

\section{RESULTS ON THE LMJ-PETAL FACILITY}

The first experimental campaign on PETAL has been conducted with the beam energy varying from 90 to $425 \mathrm{~J}$ at wavelength $1.053 \mu \mathrm{m}$ and pulse duration varying from 0.6 to $1 \mathrm{ps}$. The maximum laser intensity was in the range $10^{18}-10^{19} \mathrm{~W} / \mathrm{cm}^{2}$. The laser was focused on the target surface in a spot of about 50- $\mu \mathrm{m}$-diameter full width at half maximum (FWHM). Several types of targets were deployed: mainly $20-\mu \mathrm{m}$ gold foils for testing ion acceleration but also thicker targets (up to $2 \mathrm{~mm}$ ) for alignment or for $\mathrm{X}$-ray generation and with different materials (tungsten, plastic, aluminium, or copper). The EMP has been measured in all shots with four Prodyn probes placed at a distance of $4 \mathrm{~m}$ from the target inside the chamber and covering the frequency range up to $5 \mathrm{GHz}$ : RB50 up to $0.9 \mathrm{GHz}$ (magnetic horizontal polarization), RB270 up to $2.4 \mathrm{GHz}$ (magnetic horizontal and vertical polarization), and RB230 up to $5 \mathrm{GHz}$ (magnetic horizontal polarization). An additional (a)

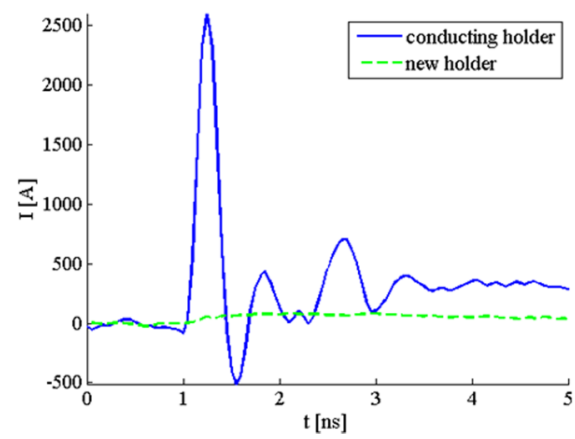

(b)

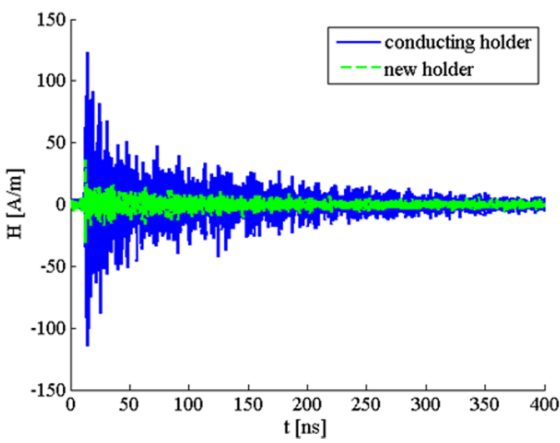

(c)

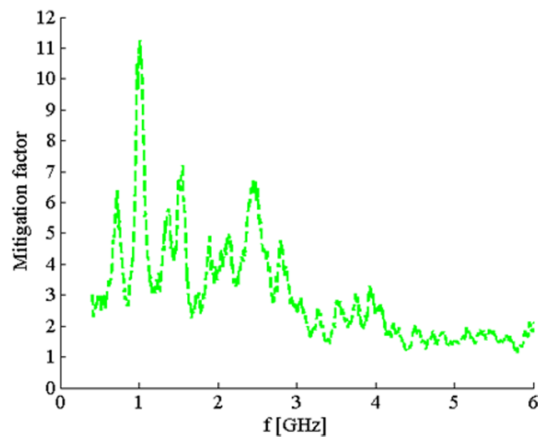

FIG. 2. Results of the LULI campaign: (a) Time dependence of the discharge current in the reference conducting holder (blue solid line) and in the new resistive holder (green dashed line). (b) Time dependence of the magnetic field measured at a distance of $54 \mathrm{~cm}$ from the target for the reference conducting holder (blue solid line) and for the new holder (green dashed line). (c) Mitigation factor for the magnetic field obtained with the new holder. 


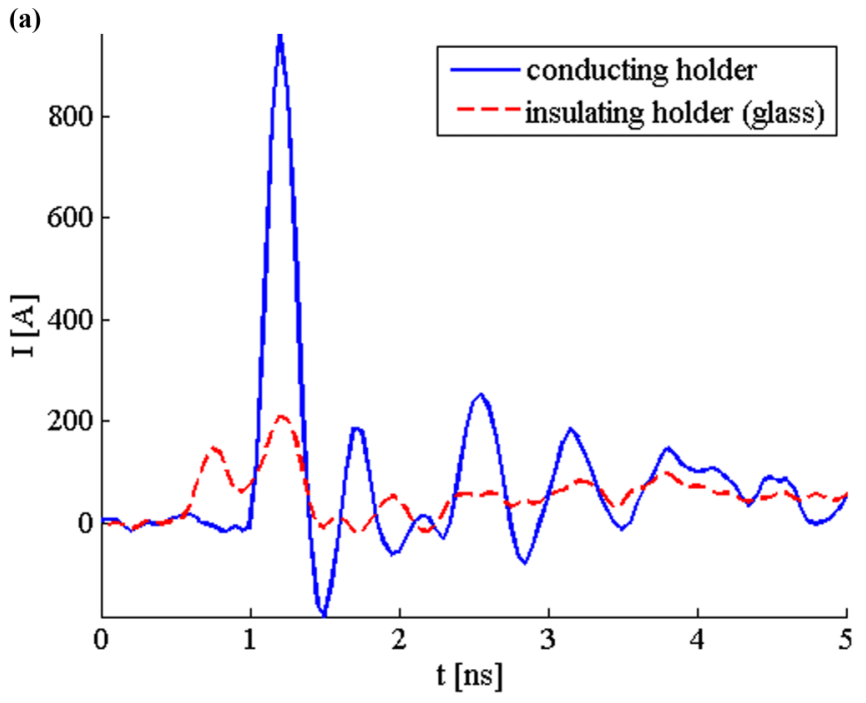

(b)

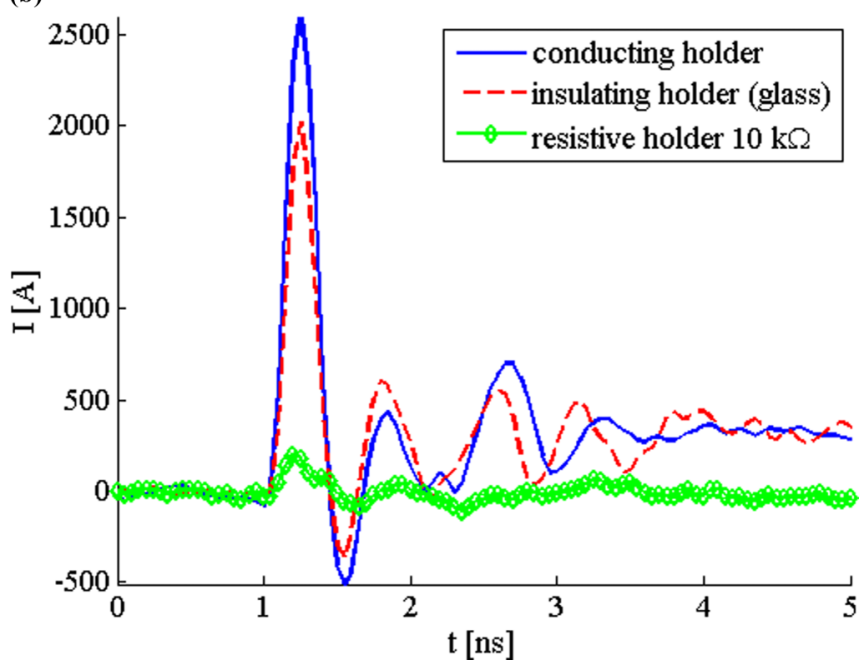

FIG. 3. Time dependence of the discharge current in the LULI experiment for low laser intensity (a) and high laser intensity (b) shots with a reference conducting holder (blue solid line), an insulating holder (red dashed line), and a resistive $10-\mathrm{k} \Omega$ holder (green diamonds).

"blind" probe surrounded by a metallic capsule was used to measure a noise level. A photo of the B-dot mount is shown in Fig. 4.

The EMP emission inside the LMJ-PETAL experimental chamber has been simulated with a set of numerical tools described in Ref. [9]. The last simulation step, which models the electromagnetic field propagation inside the entire experimental chamber, is performed with the 3D PIC code SOPHIE [12] on the CEA/DAM TERA-1000 cluster with 2048 processors. This code solves in a self-consistent way the Maxwell's equations for the propagation of electromagnetic fields in vacuum and in solid materials with realistic boundary conditions (perfect conductor, dielectric, magnetic materials) along with the relativistic dynamic equations for the particle propagation in vacuum. The mesh shown in Fig. 5 is composed of 15 billion cells and 100 million macroparticles representing hot electrons that are ejected from the target. The

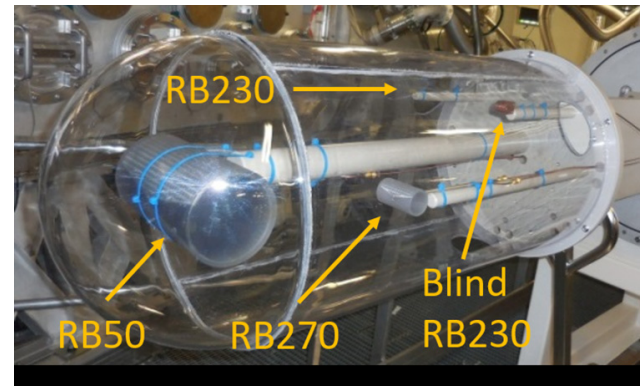

FIG. 4. Photo of B-dots probes (case of horizontal magnetic polarization) mounted on an diagnostic window in the LMJ target chamber. The B-dot holder is horizontally oriented in the chamber.

code calculates the discharge current through the target holder and electromagnetic field at the place of EMP diagnostics at $4 \mathrm{~m}$ from the target chamber center (TCC). For PETAL beam energies varying from 100 to $1000 \mathrm{~J}$, a tantalum 2-mm-thick target with $10 \mathrm{~mm}$ diameter was mounted on a $15-\mathrm{cm}$-high standard conducting holder, in order to evaluate a maximum radiated field.

The results of these PETAL experiments are presented in Fig. 6 and compared to the simulation results. Only signals from the RB270 probe are presented here because both polarizations are measured and the EMP is maximal in this frequency range for the electric field horizontal polarization. The measured peak amplitude of the magnetic field $H_{m}$ is converted into the electric field $E_{m}$ by multiplying it by the vacuum impedance $Z_{0}=377 \Omega$. The far-field approximation $E_{m}=Z_{0} H_{m}$ is justified at a distance of $4 \mathrm{~m}$ from the EMP source, which is ten times larger than the characteristic wavelength. The measurement uncertainty, represented by the error bars, contains two components. The primary part of about $\pm 40 \%$ corresponds to the shot-to-shot variation; a secondary part of $\pm 10 \%$ corresponds to the intrinsic uncertainty of the measurement channel, deduced from the noise level recorded by the blind probe. Thus, the main source of uncertainty is the reproducibility of the EMP emission. The latter is due to poorly controlled laser parameters (spot size, contrast, pulse duration) and, to a lesser extent, to target parameter variations

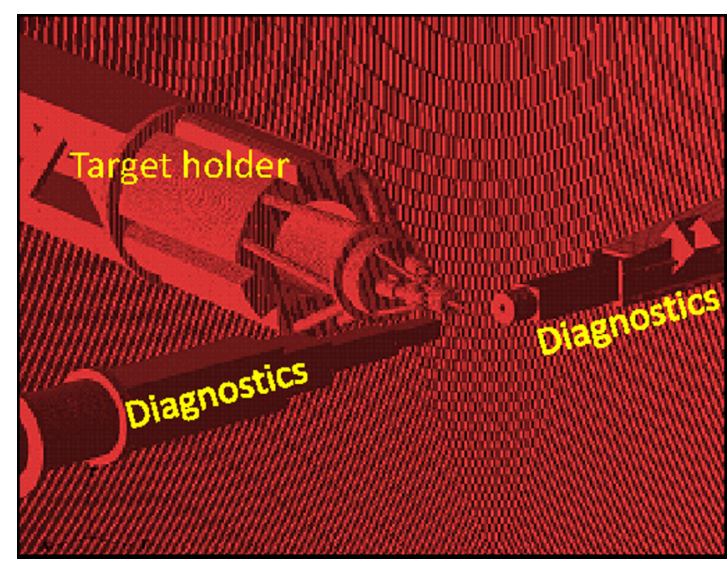

FIG. 5. Mesh for numerical simulation of EMP emission in the PETAL experiment with SOPHIE code. It includes 15 billion cells distributed in a volume of $550 \mathrm{~m}^{3}$. 

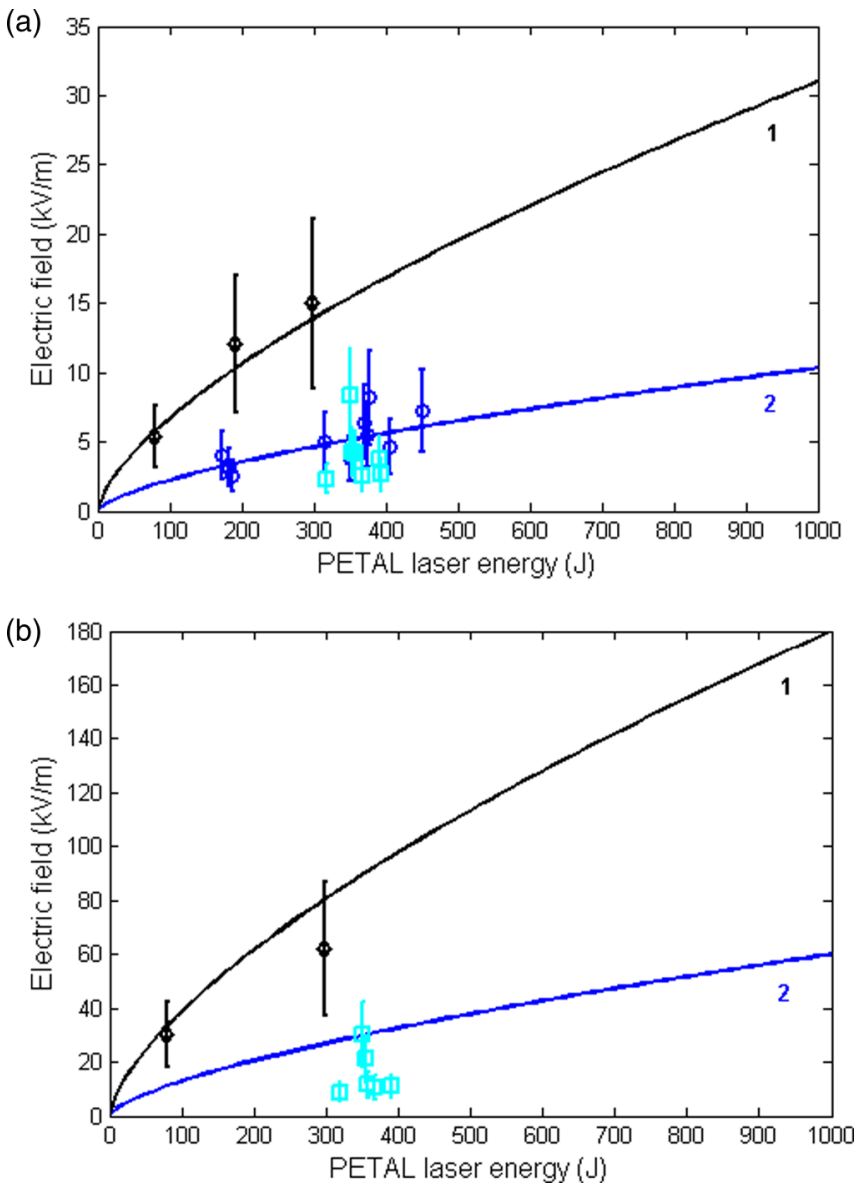

FIG. 6. Electric field maximum amplitude $E_{m}$ of vertical (a) and horizontal (b) polarization detected in PETAL experiments for different types of holder (diamonds, squares, circles) and obtained in numerical simulations performed with the code SOPHIE for a reference conducting holder (black line 1). Three sets of experimental results are presented: Shots with the reference conducting holder (black diamonds), shots with the new holder (dark blue circles), and shots with a dielectric holder (light blue squares). Blue line (2) shows the simulation results divided by a factor of 3 . The signals are detected with RB270 probes in the frequency range $0.15-2.4 \mathrm{GHz}$.

(thickness, size, materials). The $\pm 40 \%$ confidence interval corresponds to twice the experimental standard deviation of several measurements for similar shots.

Numerical simulations predict that a charge of about $1 \mu \mathrm{C}$ is ejected from the target, which is charged to a potential of about $10 \mathrm{MV}$ for a laser pulse energy of $500 \mathrm{~J}$. The numerical simulation results give a discharge current intensity of about $10 \mathrm{kA}$, which leads to an EMP field amplitude of about $100 \mathrm{kV} / \mathrm{m}$ at a distance of $4 \mathrm{~m}$ from the target. The measured EMP signal is in good agreement with the simulation conducted for a standard metallic holder. The vertical electric component is five times smaller than the horizontal one. Therefore, the electric field is horizontally polarized, parallel to the holder orientation. That proves that the target holder, which acts as an antenna, is the dominant EMP source in the considered frequency range. The EMP amplitude increases with the laser energy in a power 0.66. This power law can be explained as follows. The total number of hot electrons $N_{h}$ produced during the laser target-interaction is proportional to the absorbed laser energy $\eta E_{\text {las }}$ and inversely proportional to the hot electron temperature, $N_{h} \propto \eta E_{\text {las }} / T_{h}$. The hot electrons temperature in the domain of interest scales as the square root of the laser intensity [16], while the absorption efficiency $\eta$ scales as power $1 / 4$ of the laser intensity [17]. Since in our experiment the laser intensity is proportional to the laser energy, a scaling with power law 0.75 is expected, which is quite close to the measured one.

The new target holder with the EMP mitigation system reduces the peak electric field by a factor of 3 in the $\mathrm{GHz}$ frequency bandwidth. Figure 6 shows that the performances of this new holder are quite similar to the dielectric holder. As said previously, there is, however, a significant difference: in the latter case a significant electric charge remains for a long time on target, thus creating a risk of uncontrolled electrical breakdown. With the new holder, EMP is mitigated without risk of breakdown. We expect that EMP in a 1-kJ PETAL shot will be on a level of $60 \mathrm{kV} / \mathrm{m}$, that is, comparable to a 300-J shot with the reference conducting target holder.

\section{JOINT SHOTS}

The PETAL beam performance has also been tested in combined shots with LMJ. PETAL beam of energy of $360 \mathrm{~J}$ at wavelength $1.053 \mu \mathrm{m}$ and pulse duration of about $0.8 \mathrm{ps}$ was deployed on a $50-\mu \mathrm{m}$-thick gold foil for the proton radiography of a plasma created with four LMJ quadruplets. The total energy of these four quadruplets is about $40 \mathrm{~kJ}$ at wavelength of $351 \mathrm{~nm}$ and $5 \mathrm{~ns}$ in duration. They irradiated two $\mathrm{CH}$ discs $250 \mu \mathrm{m}$ thick and $5 \mathrm{~mm}$ in diameter attached to aluminum support. The distance between the $\mathrm{CH}$ disks was $8 \mathrm{~mm}$ and the PETAL target was protected by a $1-\mu \mathrm{m}$ gold foil placed at a distance of $1 \mathrm{~mm}$. Both targets were placed on the same target holder, which was equipped with the EMP mitigation system - a glass capillary filled with a resistive gel and a ferrite bead. The distance between the two target centers was $30 \mathrm{~mm}$. The target setup is shown in Fig. 7. The PETAL beam was delayed with respect to the LMJ beams by $15-27 \mathrm{~ns}$ in order to evaluate the plasma temporal evolution.

Figure 8 presents the raw oscilloscope traces from four joint shots with a PETAL pulse delay increasing from 15 to $27 \mathrm{~ns}$. The EMP measurements of the peak electric field in these shots are summarized in Fig. 9 in function of time delay between the LMJ and PETAL pulses. As the PETAL energy for these four joint shots varies from 315 to $395 \mathrm{~J}$, a normalized value called "equivalent electric field" is shown at the $y$ axis, which is defined as

$$
E_{\text {eq }}=E_{m}\left(360 / E_{\text {las }}\right)^{0.66},
$$

where $E_{m}$ is the measured maximum electric field. The power index 0.66 corresponds to the EMP amplitude scaling with the laser energy observed in the simulation and also in the measurement (see Fig. 6). The LMJ energy also varies from 15 to $50 \mathrm{~kJ}$ (see boxed values in Fig. 9) but the EMP is known to be quite insensitive to long pulse laser energy variations [18].

Unexpectedly, the signals appears to be strongly dependent on the time delay between the PETAL and LMJ shots (Figs. 8 and 9). For time delays smaller than $20 \mathrm{~ns}$, the EMP due 


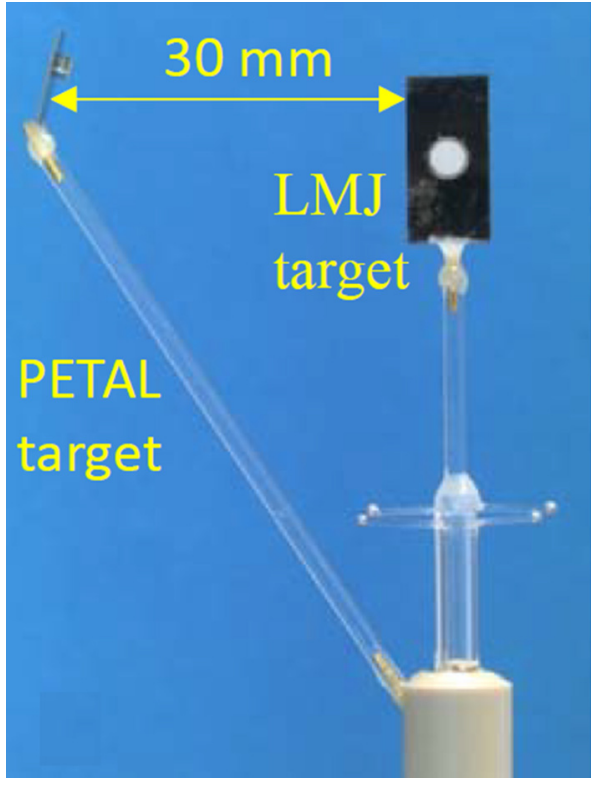

FIG. 7. Photo of the target used in the combined LMJ-PETAL laser shots. The distance between the two targets is $30 \mathrm{~mm}$.

to the PETAL interaction is suppressed, and one observes only signal originated from the LMJ interaction. Conversely, for longer delays, the EMP level is quite similar to the one measured in a PETAL single shot. The mitigation effect is even stronger than the one achieved with the new holder.

While this phenomenon is not yet understood completely for the moment, the following explanations can be proposed. The cumulated intensity of LMJ beams on aluminum targets in this experiment was about $10^{14} \mathrm{~W} / \mathrm{cm}^{2}$. Strong $\mathrm{x}$-ray emissions and copious hot electrons with characteristic energies $20-50 \mathrm{keV}$ have been generated due to the parametric instabilities. The capacitance of the LMJ target is relatively large, on the order of a few $\mathrm{pF}$, and a significant number

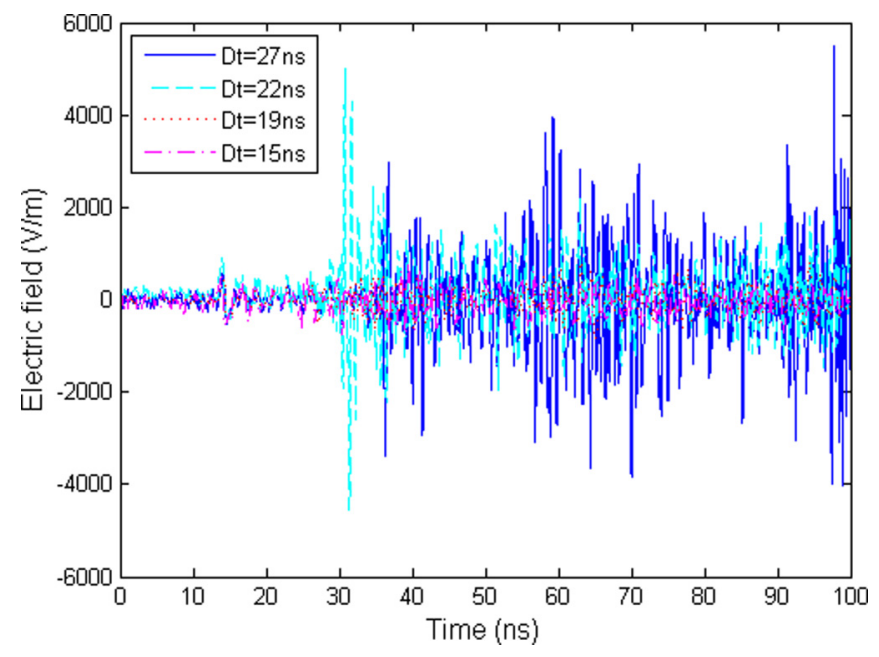

FIG. 8. Raw signals detected with the RB270 probe in the frequency range $0.15-2.4 \mathrm{GHz}$ at magnetic horizontal polarization. Four trances are shown for the time delays of $15 \mathrm{~ns}$ (pink dash-dotted line), $19 \mathrm{~ns}$ (red dotted line), $22 \mathrm{~ns}$ (light blue dashed line), and $27 \mathrm{~ns}$ (dark blue solid line).

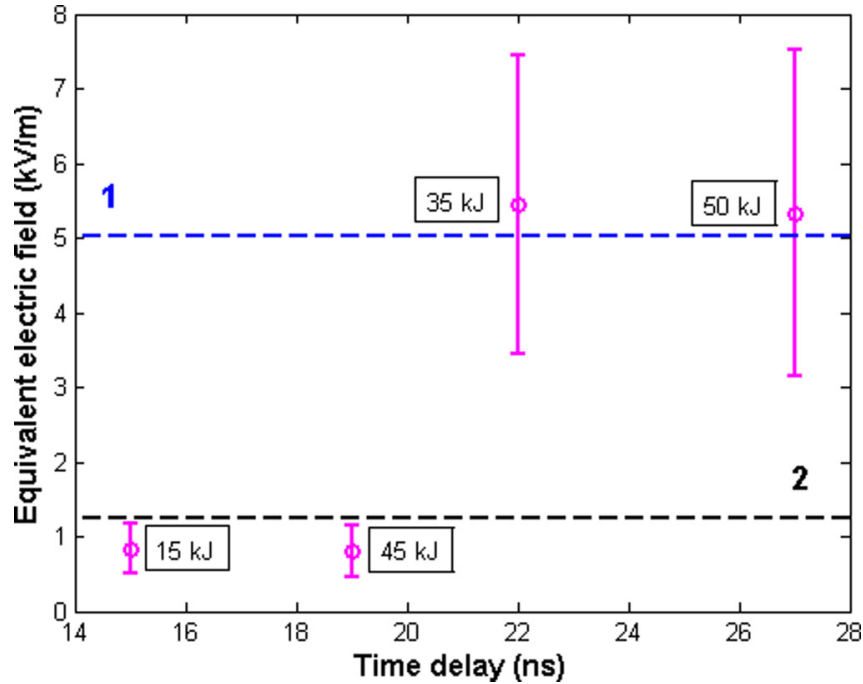

FIG. 9. Dependence of the equivalent electric field amplitude $E_{\text {eq }}$ (RB270 probe, vertical polarization) on the time delay between LMJ beams and the PETAL beam, for the four joint shots (magenta circles) with identical targets. The error bars correspond to measurement uncertainties described in the text. The dashed blue line (1) is the expected electric field level for PETAL-alone shots at $360 \mathrm{~J}$ (shown in Fig. 6) and the dashed black line (2) is the mean electric field level measured for $20 \mathrm{~kJ}$ LMJ-alone shots on the same target. The energy levels for LMJ beams are presented in boxed values.

of hot electrons $\approx 1 \mu \mathrm{C}$ can be ejected while charging the target positively to potential $\approx 100 \mathrm{kV}$. These electrons can accumulate on the PETAL's target and charge it negatively. However, as the energy of these electrons is limited to a maximum of a few hundred keV, they can charge PETAL target only to the same potential as the potential of the LMJ target. That is much smaller than the 10-MV potential created in the separate PETAL shots. Correspondingly, it seems unlikely that the electrons originating from the LMJ target can deposit a charge comparable to a $\mu \mathrm{C}$-scale charge produced in the PETAL shots.

Another possible explanation is related to the $\mathrm{x}$-ray emission from the LMJ target. A few percent of the LMJ laser energy is converted in $x$ rays, that is about $1-3 \mathrm{~kJ}$. The $\mathrm{x}$-ray spectrum measured during this experiment corresponds to a Planckian distribution with a temperature about $100 \mathrm{eV}$. This corresponds to an emission of $N_{\mathrm{ph}} \approx 10^{20}$ photons. They can ionize the residual gas (nitrogen) in the target chamber and vaporize the protective thin gold foil placed near the PETAL target. The ionization cross section of nitrogen atoms [19] with $100-\mathrm{eV}$ photons is $\sigma_{\mathrm{ph}} \approx 10^{-18} \mathrm{~cm}^{2}$. The ionization fraction $n_{e} / n_{\text {at }}$ can be estimated as

$$
n_{e} / n_{\mathrm{at}}=1-\exp \left(-\sigma_{\mathrm{ph}} F_{\mathrm{ph}}\right),
$$

where $F_{\mathrm{ph}}=N_{\mathrm{ph}} / 4 \pi R^{2}$ is the photon flux at a distance $R$ from the LMJ target. Correspondingly, at $3 \mathrm{~cm}$ from the LMJ target, at the position of the PETAL target, the photon flux is about $10^{18} \mathrm{~cm}^{-2}$, which gives an ionization fraction of about $50 \%$. Thus, the plasma environment created with the $\mathrm{x}$-ray emission may suppress the EMP emission from the PETAL target if 
the electron plasma frequency is larger than the characteristic frequency of EMP.

These estimates are confirmed by numerical simulations with the Monte Carlo code PENELOPE [20]: $10^{20}$ photons with thermal spectrum corresponding to a $100-\mathrm{eV}$ temperature were emitted isotropically from a point-like source in nitrogen gas. Nitrogen atoms were fully ionized in a sphere of a radius greater than $3 \mathrm{~cm}$. The residual air pressure in the chamber in this experiment was $5 \times 10^{-6}$ mbar, which corresponds to a nitrogen atomic density $n_{\text {at }} \approx 1 \times 10^{11} \mathrm{~cm}^{-3}$. For a full ionization, the free electron density at the position of the PETAL target is expected to be 7 times the nitrogen atomic density, that is, $n_{e} \approx 7 \times 10^{11} \mathrm{~cm}^{-3}$, which corresponds to a plasma frequency of $7.5 \mathrm{GHz}$. This is sufficient to suppress the EMP propagation emitted from the PETAL target.

It is not clear for the moment why strong EMP emission reappears for delays longer than $20 \mathrm{~ns}$. The plasma recombination time is in a $\mu$ s timescale. It is much longer than the observed EMP suppression time, but plasma recombination might be accelerated by contact with a massive LMJ target and remnants of the gold film.

Our best hypothesis is that, for delays shorter than plasma recombination time, the EMP resulting from the PETAL interaction is shielded in the ambient plasma created by $\mathrm{x}$-ray ionization of the residual air and does not propagate far away. Conversely, for longer time delays, the LMJ shot memory is lost and the PETAL target produces the same EMP as when it is standing alone. Unfortunately, due to limited diagnostics deployed during these shots, we were not able to validate this hypothesis. Further experiments and simulations have to be conducted in order to conclude about the mechanisms of EMP suppression. If confirmed, this phenomenon opens another efficient way for EMP control in high-power laser experiments.

\section{CONCLUSION}

In conclusion, a LMJ-PETAL target holder has been designed by numerical simulations and validated in short-pulse laser experiments with energy up to $400 \mathrm{~J}$. A reduction by a factor of 3 of the radiated electromagnetic field is observed in a $3-\mathrm{GHz}$ bandwidth. Numerical simulations are in good agreement with the experiment and confirm that the target holder is the major EMP source. Moreover, a spectacular phenomenon is observed in LMJ-PETAL coupling shots. For time delays smaller than 20 ns, we observe a strong EMP suppression from the PETAL shot. This phenomenon opens another efficient way for the EMP control in high-power laser experiments. It will be further studied in numerical simulations and experiments.

\section{ACKNOWLEDGMENTS}

This study has been carried out with financial support from the French National Agency of Research, Contract No. ANR-10-EQPX-0048 and Programme IdEx BordeauxLAPHIA (ANR-10-IDEX-03-02). The PETAL project has been performed by the commissariat à l'énergie atomique et aux énergies alternatives ("maître d'oeuvre") under the financial auspices of the New Aquitaine Region in France ("maître d'ouvrage," project owner), the French Government, the European Union, and the scientific support from the Institute of Lasers et Plasmas.
[1] A. Casner, T. Caillaud, S. Darbon, A. Duval, I. Thfouin, J. P. Jadaud, J. P. LeBreton, C. Reverdin, B. Rosse, R. Rosch, N. Blanchot, B. Villette, R. Wrobel, and J. L. Miquel, LMJ/PETAL laser facility: Overview and opportunities for laboratory astrophysics, High Energy Density Phys. 17, 2 (2015).

[2] M. Nicolaizeau and P. Vivini, LMJ status: Second bundle commissioning and assessment of first years of service, Proc. SPIE 10084, 1008402 (2017).

[3] V. Denis, V. Beau, L. Le Deroff, L. Lacampagne, T. Chies, X. Julien, E. Bordenave, C. Lacombe, S. Vermersch, and J.-P. Airiau, The Laser Megajoule Facility: Laser performances and comparison with computational simulation, Proc. SPIE 10084, 100840I (2017).

[4] N. Blanchot G. Béhar, J. C. Chapuis, C. Chappuis, S. Chardavoine, J. F. Charrier, H. Coïc, C. Damiens-Dupont, J. Duthu, P. Garcia, J. P. Goossens, F. Granet, C. Grosset-Grange, P. Guerin, B. Hebrard, L. Hilsz, L. Lamaignere, T. Lacombe, E. Lavastre, T. Longhi, J. Luce, F. Macias, M. Mangeant, E. Mazataud, B. Minou, T. Morgaint, S. Noailles, J. Neauport, P. Patelli, E. Perrot-Minnot, C. Present, B. Remy, C. Rouyer, N. Santacreu, M. Sozet, D. Valla, and F. Laniesse, 1.15 PW-850 J compressed beam demonstration using the PETAL facility, Opt. Express 25, 16957 (2017).

[5] M. J. Mead, D. Neely, J. Gauoin, R. Heathcote, and P. Patel, Electromagnetic pulse generation within a petawatt laser target chamber, Rev. Sci. Instr. 75, 4225 (2004).
[6] C. G. Brown Jr., A. Throop, D. Eder, and J. Kimbrough, Electromagnetic pulses at short-pulse laser facilities, J. Phys.: Conf. Ser. 112, 032025 (2008).

[7] C. G. Brown Jr., E. Bond, T. Clancy, S. Dangi, D. C. Eder, W. Fergusson, J. Kimbrough, and A. Throop, Assessment and mitigation of electromagnetic pulse (EMP) impacts at short-pulse laser facilities, J. Phys.: Conf. Ser. 244, 032001 (2010).

[8] D. C. Eder, R. W. Anderson, D. S. Bailey, P. Bell, D. J. Benson, A. L. Bertozzi, W. Bittle, D. Bradley, C. G. Brown, T. J. Clancy, H. Chen, J. M. Chevalier, P. Combis, L. Dauffy, C. S. Debonnel, M. J. Eckart, A. C. Fisher, A. Geille, V. Y. Glebov, J. Holder, J. P. Jadaud, O. Jones, T. B. Kaiser, D. Kalantar, H. Khater, J. Kimbrough, A. E. Koniges, O. L. Landen, B. J. MacGowan, N. D. Masters, A. MacPhee, B. R. Maddox, M. Meyers, S. Osher, R. Prasad, D. Raffestin, J. Raimbourg, V. Rekow, C. Sangster, P. Song, C. Stoeckl, M. L. Stowell, J. M. Teran, A. Throop, R. Tommasini, J. Vierne, D. White, and P. Whitman, Assessment and mitigation of radiation, EMP, debris and shrapnel impacts at megajoule-class laser facilities, J. Phys.: Conf. Ser. 244, 032018 (2010).

[9] J.-L. Dubois, F. Lubrano-Lavaderci, D. Raffestin, J. Ribolzi, and J. Gazave, A. Compant La Fontaine, E. d'Humières, S. Hulin, P. Nicolaï, A. Poyé, and V. T. Tikhonchuk, Target charging in short-pulse-laser-plasma experiments, Phys. Rev. E 89, 013102 (2014). 
[10] A. Poyé, S. Hulin, M. Bailly-Grandvaux, J.-L. Dubois, J. Ribolzi, D. Raffestin, M. Bardon, F. Lubrano-Lavaderci, E. d'Humières, J. J. Santos, P. Nicolaï, and V. T. Tikhonchuk, Physics of giant electromagnetic pulse generation in short-pulse laser experiments, Phys. Rev. E 91, 043106 (2015).

[11] A. Poyé, J.-L. Dubois, F. Lubrano-Lavaderci, E. d'Humières, M. Bardon, S. Hulin, M. Bailly-Grandvaux, J. Ribolzi, D. Raffestin, J. J. Santos, P. Nicolaï, and V. T. Tikhonchuk, Dynamic model of target charging in short laser pulse interactions, Phys. Rev. E 92, 043107 (2015).

[12] O. Cessenat, SOPHIE, a high performance code for travelling waves and charged particles, CHOCS 44, 25 (2013).

[13] F. Lubrano, M. Bardon, J.-L. Dubois, and S. Champeaux, Dispositif d'atténuation de l'IEM générée dans une installation laser de puissance, INPI Patent 14 59941, 2014.

[14] http://www.celia.u-bordeaux.fr/spip.php?article254.

[15] https://portail.polytechnique.edu/luli/fr/installations/luli2000.

[16] S. C. Wilks, W. L. Kruer, M. Tabak, and A. B. Langdon, Absorption of Ultra-Intense Laser Pulses, Phys. Rev. Lett. 69, 1383 (1992).

[17] R. P. J. Town, C. Chen, L. A. Cottrill, M. H. Key, W. L. Kruer, A. B. Langdon, B. F. Lasinski, R. A. Snavely, C. H. Still, M.
Tabak, D. R. Welch, and S. C. Wilks, Simulations of electron transport for fast ignition using LSP, Nucl. Instr. Meth. A 544, 61 (2005).

[18] F. Consoli, V. T. Tikhonchuk, M. Bardon, P. Bradford, D. C. Carroll, J. Cikhardt, M. Cipriani, R. J. Clarke, T. E. Cowan, C. N. Danson, R. De Angelis, M. De Marco, J.L. Dubois, B. Etchessahar, A. Laso Garcia, D. I. Hillier, A. Honsa, W. Jiang, V. Kmetik, J. Krasa, Y. Li, F. Lubrano, P. McKenna, J. Metzkes-Ng, A. Poyé, I. Prencipe, P. Raczka, R. A. Smith, R. Vrana, N. C. Woolsey, E. Zemaityte, Y. Zhang, Z. Zhang, B. Zielbauer, and D. Neely, Laser produced electromagnetic pulses: Generation, detection, and mitigation, High Power Laser Sci. Eng. 8, e22 (2020).

[19] Y. B. Zel'dovich and Y. P. Raiser, in Physics of Shock Waves and High-Temperature Hydrodynamic Phenomena, edited by W. D. Hayes and R. F. Probstein (Academic Press, New York, 1966), Vol. 1, Chaps. V, VI.

[20] J. Sempau, E. Acosta, J. Baro, J. M. Fernandez-Varea, and F. Salvat, An algorithm for Monte Carlo simulation of the coupled electron-photon transport, Nucl. Instr. Meth. B 132, 377 (1997). 